Portland State University

PDXScholar

$1-2015$

\title{
High-Resolution Bottom-Loss Estimation Using the Ambient-Noise Vertical Coherence Function
}

\author{
Lanfranco Muzi \\ Portland State University \\ Martin Siderius \\ Portland State University, siderius@pdx.edu \\ Jorge E. Quijano \\ University of Victoria \\ Stan E. Dosso \\ University of Victoria
}

Follow this and additional works at: https://pdxscholar.library.pdx.edu/ece_fac

Part of the Acoustics, Dynamics, and Controls Commons

Let us know how access to this document benefits you.

\author{
Citation Details \\ Muzi, L., Siderius, M., Quijano, J. E., \& Dosso, S. E. (2015). High-resolution bottom-loss estimation using \\ the ambient-noise vertical coherence function. The Journal of the Acoustical Society of America, 137(1), \\ 481-491.
}

This Article is brought to you for free and open access. It has been accepted for inclusion in Electrical and Computer Engineering Faculty Publications and Presentations by an authorized administrator of PDXScholar. Please contact us if we can make this document more accessible: pdxscholar@pdx.edu. 


\title{
High-resolution bottom-loss estimation using the ambient-noise vertical coherence function
}

\author{
Lanfranco Muzi ${ }^{\mathrm{a})}$ and Martin Siderius \\ Department of Electrical and Computer Engineering, Portland State University, 1900 Southwest 4th Avenue, \\ Portland, Oregon 97201 \\ Jorge E. Quijano and Stan E. Dosso \\ School of Earth and Ocean Sciences, University of Victoria, 3800 Finnerty Road, Victoria, \\ British Columbia V8P 5C2, Canada
}

(Received 14 February 2014; revised 15 September 2014; accepted 12 November 2014)

\begin{abstract}
The seabed reflection loss (shortly "bottom loss") is an important quantity for predicting transmission loss in the ocean. A recent passive technique for estimating the bottom loss as a function of frequency and grazing angle exploits marine ambient noise (originating at the surface from breaking waves, wind, and rain) as an acoustic source. Conventional beamforming of the noise field at a vertical line array of hydrophones is a fundamental step in this technique, and the beamformer resolution in grazing angle affects the quality of the estimated bottom loss. Implementation of this technique with short arrays can be hindered by their inherently poor angular resolution. This paper presents a derivation of the bottom reflection coefficient from the ambient-noise spatial coherence function, and a technique based on this derivation for obtaining higher angular resolution bottomloss estimates. The technique, which exploits the (approximate) spatial stationarity of the ambientnoise spatial coherence function, is demonstrated on both simulated and experimental data.
\end{abstract}

(C) 2015 Acoustical Society of America. [http://dx.doi.org/10.1121/1.4904508]

[JAC]

Pages: 481-491

\section{INTRODUCTION}

Underwater acoustic-propagation models rely on accurate information about the acoustic properties of the two boundaries of the ocean waveguide: the sea surface and the bottom. Especially for models based on ray tracing, this information can be in the form of the reflection loss as a function of grazing angle and frequency. The dependence of the bottom reflection loss (hereafter, also referred to as "bottom loss") on the grazing angle and on the frequency of the incident wave is determined by the thickness and physical properties of the layers, which can vary dramatically within a few hundred meters in lateral directions. ${ }^{1,2}$ The bottom properties are costly and difficult to measure directly in situ (e.g., by collection and analysis of seabed cores), ${ }^{3,4}$ and are typically obtained either from existing environmental databases (when available), or by geoacoustic inversion of measured acoustic data, with the latter method potentially capable of providing adequate spatial resolution for accurate propagation modeling.

Perhaps the most widely employed methodology for geoacoustic inversion has, so far, been deploying acoustic sources (such as sound projectors or explosive charges-or exploiting sources of opportunity, such as ship noise) and hydrophone arrays, measuring the acoustic field, and employing model-based matched-field processing to determine the seabed properties by minimizing the mismatch between model predictions and measurement. Typical disadvantages of systems that use acoustic sources are the environmental impact and the costly deploying techniques and equipment.

\footnotetext{
a) Author to whom correspondence should be addressed. Electronic mail: muzi@pdx.edu
}

Passive systems (which only exploit acoustic sources already present in the environment) have the advantage of reduced environmental impact and can be easier to deploy and operate. The work described in this paper focuses on improving the grazing-angle resolution of bottom-loss estimates obtained from passive data collected by vertical line arrays.

Marine ambient noise generated at the surface by breaking waves, wind, and rain has received increased interest lately as an acoustic source, allowing the development of passive techniques for surveying the sea bottom, such as Harrison and Simons' technique for bottom-loss estima$\operatorname{tion}^{5-10}$ (and its extension to the investigation of bottom layering ${ }^{11,12}$ ) and the passive fathometer. ${ }^{13-17}$ Harrison and Simons' technique produces an estimate of the bottom loss, as a function of frequency and grazing angle, by beamforming ambient-noise data collected by a vertical line array of hydrophones. The resulting data could be input directly to some propagation models, or used for geoacoustic inversion to estimate seabed properties, such as sediment sound speed, density, and attenuation. ${ }^{8,9}$ However, with this technique the angular resolution of bottom-loss estimates is affected by the limited aperture of the array: All other parameters being equal, the resolution improves when the array length (and number of sensors) increases. ${ }^{18}$

Harrison and Simons' technique has been applied to data collected by moored or drifting arrays, ${ }^{11,12}$ with the latter deployment technique affording the possibility of surveying an extensive area as the array is carried by the current. The arrays were several meters long and had a flexible construction, which can make beamforming more challenging if the array shape departs from a straight vertical line during data collection ("array mismatch" error). This problem could be 
eliminated by using rigid arrays, and some authors ${ }^{7,8}$ have proposed mounting short, rigid arrays on autonomous underwater vehicles to obtain an efficient, cost-effective survey tool for seabed characterization, providing long duration at sea and coverage of extended areas with minimal demands on vessel or human resources. However, for the purpose of bottom-loss estimation, with short arrays poor angular resolution becomes a matter of concern. The consequences on the estimated bottom loss can include a shift in the location of the critical angle and, if the seabed is layered, poor definition of interference features in the computed bottom loss. ${ }^{8}$ These errors are not desirable when the estimated bottom loss is used directly in propagation models, or in an inversion scheme to estimate geoacoustic properties of the seabed.

This paper illustrates a technique for improving the angular resolution of the bottom-loss estimation. The technique was introduced by Siderius et al. in a previous publication, ${ }^{10}$ and shown in simulation to outperform conventional delay-andsum beamforming in bottom-loss estimation. Here, the theoretical treatment is expanded to include volume attenuation and variable sound speed in the water column, and results are presented from its application to measured data from several at-sea experiments. In particular, one example is shown that illustrates how contamination of the ambient-noise field by other sources (e.g., shipping noise) can alter the structure of the cross-spectral-density matrix in a way that can make the results of these bottom-loss estimation techniques less reliable.

The remainder of this paper is organized as follows. Section II summarizes Harrison and Simons' technique to obtain the bottom-loss estimates from ambient-noise beamforming. Section III presents the derivation, in the frequency-wave number domain, for obtaining the bottom power reflection coefficient from the noise spatial coherence function and analyzes the conditions under which the result holds. Section IV illustrates the implementation of the proposed technique. Section V shows the results of the technique on simulation data, investigates how the structure of the cross-spectral-density matrix influences the bottom-loss estimate, and introduces the problems that can arise when applying this technique to measured data. Section VI shows the results of the technique on experimental data collected at three different locations and employing two different arrays. Section VII summarizes the main findings of this study.

\section{BOTTOM-LOSS ESTIMATION AND BEAMFORMING}

A passive technique for estimating the bottom loss was first introduced by Harrison and Simons, ${ }^{5}$ and has proven effective in several studies. ${ }^{6,8,9}$ In this technique, the marine ambient-noise field, mainly originating from breaking waves, wind, and rain at the surface, is sampled at discrete locations in space by a vertical line array of hydrophones. The data are then beamformed to obtain estimates of the power impinging on the array from different angles. The ratio of the noise coming from the seabed to that coming from the surface (at opposite angles with respect to the horizontal) reveals the loss due to interaction with the seabed, which by definition is the bottom loss. Since this technique is the focus of this paper, this section describes in some detail its basics and how it is implemented in practice. Beamforming of a vertical line array is presented here in angle space, rather than the equivalent wave number-space treatment, as this has more intuitive appeal in the context of this technique.

For a wave front of angular frequency, $\omega$, incident upon the bottom at grazing angle, $\theta_{b}>0$ (see Fig. 1 for the definition of all geometric quantities), the bottom loss is defined as

$$
B L\left(\theta_{b}, \omega\right)=-10 \log _{10} R\left(\theta_{b}, \omega\right),
$$

where $R\left(\theta_{b}, \omega\right)$ is the plane-wave power reflection coefficient of the bottom. Harrison and Simons show that the bottom loss can be computed from an estimate, $\hat{R}\left(\theta_{b}, \omega\right)$, of the power reflection coefficient obtained from array data as the ratio of the downward and upward average beam powers

$$
\hat{R}\left(\theta_{b}, \omega\right)=\frac{\hat{B}\left(-\theta_{b}, \omega\right)}{\hat{B}\left(\theta_{b}, \omega\right)} .
$$

The average beam power, $B(\vartheta, \omega)$, at the steering angle, $\vartheta$, is defined as

$$
B(\vartheta, \omega)=\mathrm{E}\left[\mathbf{w}^{H} \mathbf{p}\left(\mathbf{w}^{H} \mathbf{p}\right)^{H}\right]=\mathbf{w}^{H} \mathrm{E}\left[\mathbf{p} \mathbf{p}^{H}\right] \mathbf{w} .
$$

(For the sake of simplicity, in this treatment, the dependence on frequency and angle will often be dropped in the right-hand side of equations.) In Eq. (3), $H$ denotes the conjugate transpose operation, $\mathrm{E}[\cdot]$ denotes expectation, and $\mathbf{w}(\vartheta, \omega)$ $=\left[w_{1}, w_{2}, \ldots, w_{M}\right]^{T}$ is the weight vector $(T$ denotes the transpose operation). Note that with the conventions defined in Fig. $1, \vartheta$ has the same value as the angle at the receiver, $\theta_{r}$. The angle $\vartheta=0$ corresponds to the array being steered toward broadside (i.e., horizontally for a vertical array), $\vartheta>0$ toward the surface, and $\vartheta<0$ toward the bottom. The vector, $\mathbf{p}(\omega)$ $=\left[p_{1}(\omega), p_{2}(\omega), \ldots, p_{M}(\omega)\right]^{T}$, where $p_{m}(\omega)=\mathscr{F}\left\{p_{m}(t)\right\}$, represents the data from the $M$ hydrophones in the array

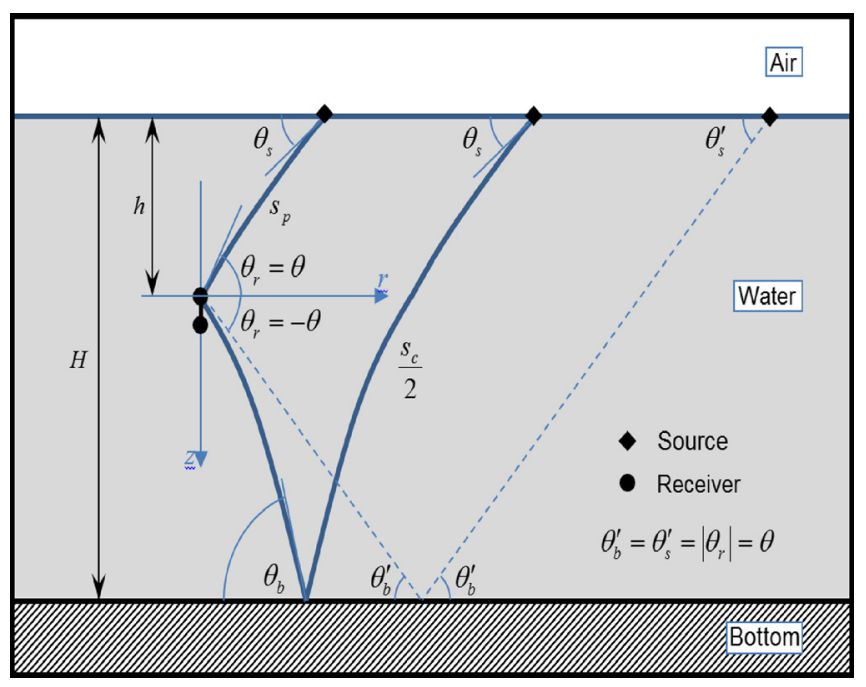

FIG. 1. (Color online) Definition of coordinate system and geometric quantities. The origin of the reference frame is at the location of sensor 1, which, in this case, is the shallowest sensor. For constant sound speed, the rays are straight lines (dashed) and $\theta_{s}^{\prime}=\theta_{b}^{\prime}=\left|\theta_{r}\right|$. The thick solid lines represent ray paths in the presence of a non-constant sound-speed profile. The same angle at the receiver, $\theta_{r}$, is considered in both cases; $s_{c}$ is the length of a surfacebottom-surface ray path. 
$(\mathscr{F}\{\cdot\}$ denotes the Fourier transform and the array-element index, $m$, increases in the same direction as the $z$-axis-i.e., the shallowest element corresponds to $m=1$ ). For the "conventional beamformer" (CBF), the weight for the $m$ th element in the array is computed as

$$
w_{m}(\vartheta, \omega)=\frac{\mathrm{w}_{m}}{\sqrt{M}} e^{-i(m-1)(\omega / c) d \sin \vartheta},
$$

where $c$ is the sound speed at the receiver, $d$ is the array inter-element spacing (assumed constant throughout the array), and $\mathrm{w}_{m}$ is a windowing coefficient (equal to 1 if no shading window is applied to the array).

The unnormalized spatial coherence matrix (or crossspectral-density matrix, hereafter also referred to as "CSD matrix"), $\mathbf{C}_{\omega}$, is defined as the expected value of the outer product $\mathbf{p}(\omega) \mathbf{p}^{H}(\omega)$,

$$
\mathbf{C}_{\omega}=\mathrm{E}\left[\mathbf{p} \mathbf{p}^{H}\right] \text {. }
$$

In real-world applications, an estimate, $\hat{\mathbf{C}}_{\omega}$, of $\mathbf{C}_{\omega}$ is obtained by averaging the outer product over $K$ snapshots as

$$
\hat{\mathbf{C}}_{\omega}=\frac{1}{K} \sum_{i=1}^{K} \mathbf{p}_{i} \mathbf{p}_{i}^{H} .
$$

This estimate is then used to replace $\mathrm{E}\left[\mathbf{p} \mathbf{p}^{H}\right]$ in Eq. (3), yielding

$$
\hat{B}(\vartheta, \omega)=\mathbf{w}^{H} \hat{\mathbf{C}}_{\omega} \mathbf{w} .
$$

Equation (2) shows that, in bottom-loss estimation, the ratio of the beamformer output power is used to estimate the power ratio of (plane) wave fronts incident upon the array from angles symmetric with respect to the horizontal. It may be useful at this point to note that in the rest of this paper the direction of propagation of a plane wave will be identified by its angle of incidence at the array (also referred to as angle at the receiver and indicated by $\theta_{r}$ in Fig. 1). Although this differs from the convention usually adopted in physics, it is common in array processing and, therefore, a more natural choice for this paper. For a plane wave propagating in direction $\theta_{r}$, the wave number, $\mathbf{\kappa}$, is defined as

$$
\mathbf{\kappa} \equiv\left[\begin{array}{ll}
\kappa_{r} & \kappa_{z}
\end{array}\right] \equiv \omega / c\left[-\cos \left(\theta_{r}\right) \quad \sin \left(\theta_{r}\right)\right],
$$

whose Cartesian components, $\kappa_{r}$ and $\kappa_{z}$, are the horizontal and vertical wave numbers, respectively; then Eq. (8) establishes the correspondence between $\theta_{r}, \kappa_{r}$, and $\kappa_{z}$.

The beamformer resolution is the ability of the beamformer to discriminate between wave fronts incident from closely spaced directions. Adopting the definition based on the Rayleigh criterion, the resolution in the vertical-wavenumber domain for a linear array is ${ }^{18}$

$$
\Delta \kappa_{z}=2 \pi / L
$$

where $\Delta \kappa_{z}$ is the distance between the two closest values of $\kappa_{z}$ that can be resolved and $L=d(M-1)$ is the total length of the array. Equation (9) shows that, for a given sensor spacing, increasing the array length (and therefore the number of sensors) results in a finer resolution in $\kappa_{z}$.

\section{DERIVATION OF THE POWER REFLECTION COEFFICIENT FROM THE NOISE SPATIAL COHERENCE FUNCTION}

\section{A. Derivation}

This section presents the derivation in the frequencywave number domain of a formula for computing the power reflection coefficient from the unnormalized spatial coherence function of the surface-generated marine noise field as recorded by the array (for the sake of brevity, "unnormalized" will be omitted in the remainder of the paper, but the spatial coherence function should always be considered in this form, unless otherwise specified). The spatial coherence function of the pressure field, $p(\mathbf{r}, t)$, between two points in space, $\mathbf{r}_{1}$ and $\mathbf{r}_{2}$, is defined as the ensemble average of the product, $p_{\mathbf{r}_{1}}(\omega) p_{\mathbf{r}_{2}}^{*}(\omega)$,

$$
C_{\omega}\left(\mathbf{r}_{1}, \mathbf{r}_{2}\right) \equiv\left\langle p_{\mathbf{r}_{1}}(\omega) p_{\mathbf{r}_{2}}^{*}(\omega)\right\rangle
$$

where * indicates complex conjugate and $p_{\mathbf{r}}(\omega)$ is the coefficient of the Fourier expansion of $p(\mathbf{r}, t)$ at angular frequency $\omega$. To make an explicit link to beamforming, in Eq. (5), element $(i, j)$ in $\mathbf{C}_{\omega}$ is given by $C_{\omega}\left(\mathbf{r}_{i}, \mathbf{r}_{j}\right)$.

Using a ray-based approach, Harrison derived a formula for the spatial coherence function of surface-generated noise in the ocean, which for the case of two hydrophones joined by a perfectly vertical line and separated by a distance $z$ is written $^{19,20}$

$$
\begin{aligned}
C_{\omega}(z)= & \int_{0}^{\pi / 2} \frac{2 \pi \sin \theta_{s} \cos \theta_{r}}{1-R_{s}\left(\theta_{s}\right) R\left(\theta_{b}\right) e^{-a s_{c}\left(\theta_{r}\right)}} \\
& \times\left\{e^{i(\omega / c) z \sin \theta_{r}} e^{-a s_{p}}+R\left(\theta_{b}\right)\right. \\
& \left.\times e^{-i(\omega / c) z \sin \theta_{r}} e^{-a\left[s_{c}\left(\theta_{r}\right)-s_{p}\left(\theta_{r}\right)\right]}\right\} d \theta_{r} .
\end{aligned}
$$

In Eq. (11), $C_{\omega}(z)$ introduces a more compact notation, $C_{\omega}(z) \equiv C_{\omega}\left(\mathbf{r}_{1}, \mathbf{r}_{2}\right)$, where it is assumed that $\mathbf{r}_{1}=(0,0)$ and $\mathbf{r}_{2}=(0, z)$-i.e., the hydrophone pair is assumed to be aligned with the $z$ axis, whose origin is at the depth of hydrophone 1 . Furthermore, $\theta_{r}, \theta_{s}$, and $\theta_{b}$ are the ray angles at the receiver, the surface, and the bottom, respectively; $s_{c}$ and $s_{p}$ are the complete and partial ray-path lengths, respectively (specifically, $s_{c}$ is the length of a surface-bottom-surface ray path), whose dependence on $\theta_{r}$ is determined by the sound-speed profile in the water column; $\omega$ is the angular frequency; $c$ is the sound speed at the receiver, and $R$ and $R_{s}$ are the bottom and surface power reflection coefficients, respectively. In general, besides the ray angle, the reflection coefficients are also a function of frequency, but for the sake of simplicity, this dependence will not be indicated explicitly. Note that $a$ is the power attenuation per unit length along the ray path. The model assumes that the hydrophones are "close," so that a single ray path and sound speed can be defined for the sensor pair (see Fig. 1 for the definition of the coordinate system and all geometric quantities).

Since $\theta_{r}$ is limited in Eq. (11) to non-negative values, by defining $\theta \equiv\left|\theta_{r}\right|$ and using Snell's law 


$$
\begin{aligned}
\theta_{s} & \equiv \sin ^{-1}\left(\frac{c}{c_{s}} \sin \theta\right), \\
\theta_{b} & \equiv \sin ^{-1}\left(\frac{c}{c_{b}} \sin \theta\right)
\end{aligned}
$$

(where $c, c_{s}$, and $c_{b}$ indicate the sound speed at the receiver, surface, and bottom, respectively), the equation can be rewritten as a function of the sole angle $\theta$,

$$
\begin{aligned}
C_{\omega}(z)= & \int_{0}^{\pi / 2} \frac{2 \pi\left(c / c_{s}\right) \sin \theta \cos \theta}{1-R_{s}(\theta) R(\theta) e^{-a s_{c}(\theta)}} \\
& \times\left\{e^{i(\omega / c) z \sin \theta} e^{-a s_{p}}+R(\theta)\right. \\
& \left.\times e^{-i(\omega / c) z \sin \theta} e^{-a\left[s_{c}(\theta)-s_{p}(\theta)\right]}\right\} d \theta .
\end{aligned}
$$

Now let

$$
k \equiv \frac{\omega}{2 \pi c} \sin \theta_{r}=\frac{\sin \theta_{r}}{\lambda},
$$

where $\lambda$ is the signal wavelength; then $\theta_{r}=\sin ^{-1}(\lambda k)$ and $0<\theta_{r}=\sin ^{-1}(\lambda k)<\pi / 2$ gives the bounds $0<k<1 / \lambda$ [note that Eq. (14) defines $k$ as a scaled vertical wave number at the receiver: $\left.k=\kappa_{z} / 2 \pi\right]$. By substituting $\theta=\sin ^{-1}(\lambda|k|)$ into Eqs. (12) and (13), and defining

$$
\frac{\tilde{G}(k)}{\lambda} \equiv \frac{2 \pi\left(c / c_{s}\right) \lambda|k|}{1-R_{S}(k) R(k) e^{-a \tilde{s}_{c}(k)}},
$$

Eq. (11) can be rewritten as

$$
\begin{aligned}
C_{\omega}(z)= & \int_{0}^{1 / \lambda} \tilde{G}(k) e^{-a \tilde{s}_{p}(k)} e^{i 2 \pi z k} d k \\
& +\int_{0}^{1 / \lambda} \tilde{G}(k) R(k) e^{-a\left[\tilde{s}_{c}(k)-\tilde{s}_{p}(k)\right]} e^{-i 2 \pi z k} d k .
\end{aligned}
$$

Now by letting

$$
\begin{aligned}
\tilde{G}_{1}(k) & \equiv \tilde{G}(k) e^{-a \tilde{s}_{p}(k)}, \\
\tilde{G}_{2}(k) & \equiv \tilde{G}(k) e^{-a\left[\tilde{s}_{c}(k)-\tilde{s}_{p}(k)\right]},
\end{aligned}
$$

and by introducing the generalized rectangle function

$$
\Pi(x) \equiv\left\{\begin{array}{lll}
0 & \text { for } & |x|>1 / 2 \\
1 & \text { for } & |x| \leq 1 / 2
\end{array}\right.
$$

Eq. (16) can be rewritten as

$$
\begin{aligned}
C_{\omega}(z)= & \int_{0}^{1 / \lambda} \tilde{G}_{1}(k) e^{i 2 \pi z k} d k+\int_{0}^{1 / \lambda} \tilde{G}_{2}(k) R(k) e^{-i 2 \pi z k} d k \\
= & \int_{-\infty}^{\infty} \tilde{G}_{1}(k) \Pi(\lambda k-1 / 2) e^{i 2 \pi z k} d k \\
& +\int_{-\infty}^{\infty} \tilde{G}_{2}(k) R(k) \Pi(\lambda k-1 / 2) e^{-i 2 \pi z k} d k \\
= & \mathscr{F}^{-1}\left\{\tilde{G}_{1}(k) \Pi(\lambda k-1 / 2)\right\} \\
& +\mathscr{F}\left\{\tilde{G}_{2}(k) R(k) \Pi(\lambda k-1 / 2)\right\} .
\end{aligned}
$$

Equation (19) states that the two addends can be expressed as a direct and an inverse Fourier transform between the $z$ and the $k$ domains. Taking the Fourier transform of both sides yields

$$
\begin{aligned}
\mathscr{F}\left\{C_{\omega}(z)\right\}= & \mathscr{F}\left\{\mathscr{F}^{-1}\left\{\tilde{G}_{1}(k) \Pi(\lambda k-1 / 2\}\right\}\right. \\
& +\mathscr{F}\left\{\mathscr{F}\left\{\tilde{G}_{2}(k) R(k) \Pi(\lambda k-1 / 2\}\right\}\right. \\
\equiv & \mathcal{F}^{+}(k)+\mathcal{F}^{-}(k) .
\end{aligned}
$$

The first addend in Eq. (20) reduces to the argument of the inner inverse Fourier transform

$$
\begin{aligned}
\mathcal{F}^{+}(k) & \equiv \mathscr{F}\left\{\mathscr{F}^{-1}\left\{\tilde{G}_{1}(k) \Pi(\lambda k-1 / 2)\right\}\right\} \\
& =\tilde{G}_{1}(k) \Pi(\lambda k-1 / 2),
\end{aligned}
$$

whereas the second addend, by applying the duality property of the Fourier transform, yields

$$
\begin{aligned}
\mathcal{F}^{-}(k) & \equiv \mathscr{\mathscr { F }}\left\{\mathscr{F}\left\{\tilde{G}_{2}(k) R(k) \Pi(\lambda k-1 / 2)\right\}\right\} \\
& =\tilde{G}_{2}(-k) R(-k) \Pi(\lambda k+1 / 2) .
\end{aligned}
$$

Equations (20)-(22) show that $\mathscr{F}\left\{C_{\omega}(z)\right\}$, the $k$-spectrum of the coherence function, is split into a portion, $\mathcal{F}^{+}(k)$, which is nonzero only for positive $k$ values, and a portion, $\mathcal{F}^{-}(k)$, which is nonzero only for negative $k$ values. $R(k)$ can now be computed as the ratio

$$
\begin{aligned}
R(k)= & \frac{\mathcal{F}^{-}(-k)}{\mathcal{F}^{+}(k)} \frac{\tilde{G}_{1}(k)}{\tilde{G}_{2}(k)}=\frac{\mathcal{F}^{-}(-k)}{\mathcal{F}^{+}(k)} e^{-a\left[2 \tilde{s}_{p}(k)-\tilde{s}_{c}(k)\right]}, \\
& k \in\left[\begin{array}{ll}
0 & \frac{1}{\lambda}
\end{array}\right],
\end{aligned}
$$

where, recalling that both $\tilde{G}(k)$ and $R(k)$ are even functions of $k, \mathcal{F}^{-}(-k)$ is given by

$$
\mathcal{F}^{-}(-k)=\tilde{G}_{2}(k) R(k) \Pi(\lambda k-1 / 2) .
$$

Note that, because of the rectangle functions in $\mathcal{F}^{+}(k)$ and $\mathcal{F}^{-}(k)$, the power reflection coefficient, $R(k)$, is defined only for $k \in\left[\begin{array}{ll}0 & 1 / \lambda\end{array}\right]$, i.e., $\theta_{r} \in\left[\begin{array}{ll}0 & \pi / 2\end{array}\right]$, which are the integration limits in Eq. (11). Since negative values of $k$ correspond to $\theta_{r}<0$ in Eq. (14)-i.e., waves reaching the hydrophones after reflection from the bottom-and positive values of $k$ correspond to $\theta_{r}>0$-i.e., reflection from the surface-the result in Eq. (23) is reminiscent of the method for estimating $R$ described by Harrison and Simons, ${ }^{5}$ which in the original reference is derived through an energy-flux argument.

If volume attenuation in the water column is neglected, Eqs. (15), (17), and (23) can be simplified by dropping the exponential factors. For a lossy medium, Eq. (23) shows that the ratio of the two halves of $\mathscr{F}\left\{C_{\omega}(z)\right\}$ must be corrected by the additional exponential factor, which takes into account volume attenuation along the partial and complete ray paths. In general, the exact form of $\tilde{s}_{c}$ and $\tilde{s}_{p}$ depends on the sound-speed profile, and given the definition in Eq. (1), this factor adds to the bottom loss a correction of $10 \log \left\{\exp \left[2 a \tilde{s}_{p}(k)\right.\right.$ $\left.\left.-a \tilde{s}_{c}(k)\right]\right\}$. However, Eq. (23) also shows that this correction 
can be minimized by positioning the array close to the bottom (a similar conclusion was reached by Arvelo, ${ }^{7}$ although in the context of a different derivation): In this case, the approximation, $2 \tilde{s}_{p} \approx \tilde{s}_{c}$, can be considered valid for most grazing angles (see Fig. 1), and the value of the exponential term approaches one.

For the special case of an isospeed water column of depth $H$, assuming the hydrophone pair is at depth $h$, the complete and partial ray-path lengths are

$$
\begin{aligned}
& \tilde{s}_{c}(k) \equiv \frac{2 H}{\lambda|k|}, \\
& \tilde{s}_{p}(k) \equiv \frac{h}{\lambda|k|},
\end{aligned}
$$

and Eq. (23) becomes

$$
R(k)=\frac{\mathcal{F}^{-}(-k)}{\mathcal{F}^{+}(k)} e^{-(2 a / \lambda|k|)(h-H)}, \quad k \in\left[\begin{array}{ll}
0 & \frac{1}{\lambda}
\end{array}\right] .
$$

\section{B. Applicability of the approach}

The conditions under which the results shown above apply are determined by the assumptions underlying Eq. (11). For this study, the most important assumption is that the acoustic field be generated by surface noise: The derivation does not make allowances for sources of a different kind. In the real world, this implies being able to acquire data when the surface noise is sufficiently high and shipping interference is negligible. The derivation also assumes that the spacing between the hydrophones whose data are being correlated is small enough to guarantee that the angle at the receiver for a given ray is the same for both hydrophones. This condition is usually well approximated for vertical line arrays, especially those used for beamforming. In the derivation, the hydrophones are also assumed to be joined by a perfectly vertical line. When this is not the case (e.g., for a tilted array), the accuracy in the estimate of $R(k)$ deteriorates, but this is not investigated in this study.

Finally, a correction is required in Eq. (11) in the proximity of boundaries. Harrison ${ }^{19}$ shows that such a correction can be safely neglected at distances from the boundaries on the order of $\lambda / \sin \theta_{c}$ (where $\theta_{c}$ is the critical angle), which, e.g., corresponds to about $1.5 \mathrm{~m}$ for $\theta_{c}=20^{\circ}$ and a $3 \mathrm{kHz}$ signal. When these conditions are met, CSD matrices produced by Eq. (11) result in estimates of $R(k)$ in excellent agreement with those produced using OASN, the noise-propagation module of OASES, ${ }^{21}$ which implements a full wave solution based on wave number integration for horizontally stratified media.

\section{ARRAY PROCESSING FOR HIGH-RESOLUTION BOTTOM-LOSS ESTIMATION}

\section{A. Technique implementation}

When working with array data, measurements are only available at the locations of the sensors, so the coherence function, $C_{\omega}(z)$, is sampled at integer multiples of $d$ along the $z$ axis, and its Fourier transform in Eq. (20) must be interpreted as a discrete Fourier transform (DFT). The resolution of the DFT in spatial wave number, $k$, increases with the number of samples available to the transform, i.e., with the number of array elements. This translates into better estimation of the seabed bottom loss, but it comes at the price of physically increasing the array length. An alternative approach is proposed here, which is based on the idea of exploiting the physical properties of $C_{\omega}(z)$, before applying the DFT.

In order to explain the technique, a first important consideration is the dependence of the coherence function on the hydrophone-pair depth, $h$. This dependence appears implicitly in Eq. (11) in the difference between $s_{c}$ and $s_{p}$, and its effect on the bottom reflection coefficient is quantified by the exponential correction factor in Eq. (23). This correction can become important at very shallow grazing angles, but this effect can be minimized by positioning the array close to the bottom.

When this is added to the conditions outlined in Sec. III B, the noise coherence function between two hydrophones depends primarily on the distance between the hydrophones, rather than their absolute position in the water column. In other words, equally spaced hydrophones have the same coherence function, regardless of their position in the array. This spatial stationarity of the marine ambientnoise field has been theoretically proved and verified against experimental data for both deep ${ }^{22,23}$ and shallow water ${ }^{19,24}$ (at sufficient distance from the waveguide boundaries). Harrison's ray treatment estimated that the spatial coherence function becomes weakly dependent on sensor depth at a distance from the waveguide boundaries on the order of a few wavelengths. ${ }^{19}$

For the CSD matrix, $\mathbf{C}_{\omega}$, the spatial stationarity of $C_{\omega}(z)$ implies that $C_{\omega}\left(\mathbf{r}_{i}, \mathbf{r}_{j}\right)=C_{\omega}\left(\mathbf{r}_{l}, \mathbf{r}_{m}\right)$ when $(i-j)=(l-m)$, i.e., besides being Hermitian by construction, the matrix is Toeplitz. Finally, the spatial stationarity also implies that $C_{\omega}(z)$ is (approximately) conjugate symmetric

$$
C_{\omega}(-z) \approx C_{\omega}^{*}(z)
$$

The Toeplitz structure of $\mathbf{C}_{\omega}$ implies that all the relevant information is contained in its first row. The elements in each of the diagonals parallel to the main diagonal are all equal and all correspond to the same value of $z$. However, the number of (repeated) elements in each diagonal decreases linearly with increasing $z$. When CBF is implemented as a matrix product, as in Eq. (7), this circumstance has an effect equivalent to applying a triangular shading window to the array. This type of window is not necessarily the most desirable for this application.

However, the results in Eqs. (23) and (27) can be used to implement an algorithm for fast, improved-resolution bottomloss (BL) estimation from array data, without the limitations imposed by Eq. (7). First, $\mathbf{C}_{\omega}$ is estimated by averaging array data over an adequate number of snapshots [see Eq. (6)]. If the field is only due to surface ambient noise, the CSD matrix is (approximately) Toeplitz and an average along the diagonals provides an estimate, $\hat{C}_{\omega}(z)$, of $C_{\omega}(z)$. Furthermore, the 
estimated $\hat{C}_{\omega}(z)$ can be extended to the negative side of $z$ according to Eq. (27), windowed as desired, and its DFT taken between $\hat{C}_{\omega}(-L)$ and $\hat{C}_{\omega}(L)$, i.e., over $2 M-1$ samples, rather than just $M$ samples. The ratio of the portions of the DFT of $\hat{C}_{\omega}(z)$ on the positive and negative sides of the $k$-axis [the discrete equivalent of Eq. (23)] provides an estimate of the power reflection coefficient, $R(k)$. No further correction is needed if the array is sufficiently close to the bottom, and $R(k)$ can then be mapped back to angle space, $\theta_{r}$, and used to estimate the BL according to Eq. (1).

Sections V and VI show how this technique can increase the angular resolution of the estimated bottom loss both in simulated and experimental shallow-water scenarios.

\section{B. Examples in simple scenarios}

The result in Eq. (27) deserves some more attention, as it may appear counterintuitive initially: For example, it does not hold for the case of a single point source. If such source were at depth, $h_{s}$, and range, $r$, from a receiver at depth, $h$ (Fig. 1 can still be used as reference), the normal-mode expression for the resulting pressure field at the receivers at a given frequency, $\omega$, would be ${ }^{25}$

$$
\begin{aligned}
& p_{\omega}(r, h) \approx A\left(h_{s}\right) \sum_{m=1}^{\infty} \psi_{m}\left(h_{s}\right) \psi_{m}(h) i H_{0}^{(1)}\left(\kappa_{m} r\right), \\
& A\left(h_{s}\right)=\frac{1}{4 \rho\left(h_{s}\right)},
\end{aligned}
$$

where $\rho$ is the water density, $\psi_{m}$ is the mode shape function, $\kappa_{m}$ is the modal wave number, and $H_{0}^{(1)}\left(\kappa_{m} r\right)$ is the Hankel function of the first kind [note that in the interest of readability, in this section, the notation is slightly different from that in Sec. III; letting $\mathbf{r}=(r, h)$, the equivalence between the two notations is given by $\left.p_{\omega}(r, h)=p_{\mathrm{r}}(\omega)\right]$.

Using three receivers positioned along a vertical line at depths $(D-d), D$, and $(D+d)$, the resulting coherence functions between the center receiver and the other two would be

$$
\begin{aligned}
C_{\omega}(d)= & p_{\omega}(r, D) p_{\omega}^{*}(r, D+d) \\
\approx & A^{2}\left(h_{s}\right) \sum_{m=1}^{\infty} \sum_{n=1}^{\infty} \psi_{m}\left(h_{s}\right) \psi_{m}(D+d) \\
& \times \psi_{n}\left(h_{s}\right) \psi_{n}(D)\left[i H_{0}^{(1)}\left(\kappa_{m} r\right)\right]^{*} H_{0}^{(1)}\left(\kappa_{n}, r\right) ; \\
C_{\omega}(-d)= & p_{\omega}(r, D) p_{\omega}^{*}(r, D-d) \\
\approx & A^{2}\left(h_{s}\right) \sum_{m=1}^{\infty} \sum_{n=1}^{\infty} \psi_{m}\left(h_{s}\right) \psi_{m}(D-d) \\
& \times \psi_{n}\left(h_{s}\right) \psi_{n}(D)\left[i H_{0}^{(1)}\left(\kappa_{m} r\right)\right]^{*} H_{0}^{(1)}\left(\kappa_{n}, r\right) .
\end{aligned}
$$

Due to the differences in the depth dependence of the mode shape functions, $\psi_{m}$, Eq. (29) does not necessarily imply that Eq. (27) would hold in this case. However, the surface noise considered in this paper is different from a single point source, and its peculiar nature gives rise to the result in Eq. (27). For instance, in the case of surface noise in an isospeed deep ocean, Cron and Sherman's model ${ }^{26,27}$ expresses the spatial coherence function as ${ }^{23}$

$$
\begin{aligned}
C_{\omega}(z)= & 2\left[\frac{\sin (2 \pi z / \lambda)}{2 \pi z / \lambda}+\frac{\cos (2 \pi z / \lambda)-1}{(2 \pi z / \lambda)^{2}}\right] \\
& -2 i\left[\frac{\cos (2 \pi z / \lambda)}{2 \pi z / \lambda}-\frac{\sin (2 \pi z / \lambda)}{(2 \pi z / \lambda)^{2}}\right],
\end{aligned}
$$

which yields the result in Eq. (27) exactly.

\section{APPLICATION TO DATA}

\section{A. Application to simulated data}

Section VI presents the results of applying the technique proposed in this paper to measured data. In order to facilitate the interpretation of those results, this section applies both this technique and Harrison and Simons' technique to CSD matrices obtained from an OASN simulation. Since the simulated environment is perfectly known, in this case, ground truth bottom loss can be obtained from the power reflection coefficient computed by a theoretical model, ${ }^{1}$ and used to judge the quality of the results.

The geoacoustic parameters for this test are shown in Table I. Two array configurations are used, with 16 and 32 hydrophones; the inter-sensor spacing is $0.18 \mathrm{~m}$ in both cases, with the shallowest hydrophone at a depth of $180 \mathrm{~m}$, i.e., at $20 \mathrm{~m}$ from the bottom, to minimize the effect of attenuation. In the remainder of this paper, the proposed technique will be referred to as "high-resolution bottom-loss estimation" (HR-BL). Figure 2 shows the BL theoretical prediction, the HR-BL, and the $\mathrm{CBF}$ estimate at $2500 \mathrm{~Hz}$, from OASN-generated CSD matrices of sizes $32 \times 32$ and $16 \times 16$. The spatial coherence function for HR-BL is estimated by averaging the elements of the CSD matrix along the diagonals parallel to the main diagonal (exploiting the Toeplitz character of the matrix); the function is then "doubled" by extension to the negative $z$ values and tapered with a Tukey window with 0.6 taper width (the same used to shade the array when computing the CBF estimate). The DFT of this extended coherence function is computed as a fast Fourier transform and the reflection coefficient is estimated as the ratio of the halves of the resulting $k$ spectrum, as indicated in Eq. (23).

For both array lengths, the HR-BL results follow more closely the theoretical prediction, particularly in the 32element case [Fig. 2(a)]. Moving from 32 to 16 elements [Fig. 2(b)], the CBF experiences a significant loss of resolution, failing to recover most of the details of the peaks. On the other hand, the result of the 16-element HR-BL processor

TABLE I. Bottom configuration for the OASN simulations. Attenuation in water is set by OASN to its lower bound; $\lambda$ is the signal wavelength.

\begin{tabular}{lcccccc}
\hline \hline & $\Delta(\mathrm{m})$ & $c_{p}(\mathrm{~m} / \mathrm{s})$ & $c_{s}(\mathrm{~m} / \mathrm{s})$ & $\rho\left(\mathrm{kg} / \mathrm{m}^{3}\right)$ & $\alpha_{c}(\mathrm{~dB} / \lambda)$ & $\alpha_{s}(\mathrm{~dB} / \lambda)$ \\
\hline Water & 200 & 1500 & 0 & 1000 & - & 0 \\
Sediment & 0.75 & 1550 & 0 & 1500 & 0.2 & 0 \\
Halfspace & $\infty$ & 1600 & 0 & 2000 & 0.15 & 0 \\
\hline \hline
\end{tabular}



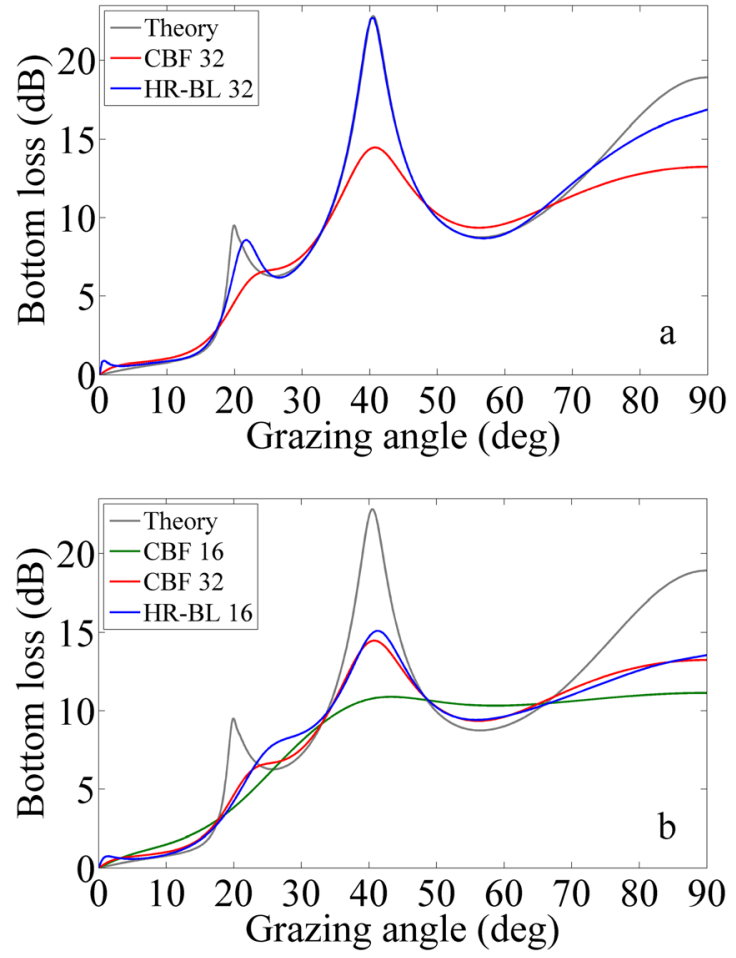

FIG. 2. (Color online) Estimated bottom loss at $2500 \mathrm{~Hz}$ from OASN data for the environment in Table I. (a) Ground truth from theoretical bottom loss ("Theory"), HR-BL processor and CBF using $32 \times 32$ CSD matrices produced by OASN. (b) Same as in (a), but using $16 \times 16$ matrices (the CBF 32 curve is repeated to facilitate a direct comparison). In both cases, when using the same number of physical sensors, the HR-BL curve is closer to the theoretical prediction than the CBF curve over almost the entire angular range. Note the significant degradation of the $\mathrm{CBF}$ when moving from 32 to 16 elements, and how the HR-BL 16 curve is very close to the CBF 32 curve. is very close to the longer CBF over almost the entire angular range (except between the peaks). Results in Sec. VI confirm that a 16-element HR-BL processor can perform at a level comparable to a 32-element physical array when applied to measured data.

To further highlight the benefits of HR-BL processing over CBF, Fig. 3 shows the bottom loss estimated over the frequency range $25-4166 \mathrm{~Hz}$ for the same OASN data used in Fig. 2, this time using a Hanning taper (the Tukey taper used in Fig. 2 can be too "aggressive" at low frequencies), and the pixel-by-pixel error between the values predicted by the theoretical model and the estimated ones. Although the Hanning taper does not maximize the performance of either technique in the upper part of the frequency range, the HR$\mathrm{BL}$ processor is closer to the theoretical prediction along the ridge peaks and performs particularly well at the lower frequencies.

\section{B. More on the Toeplitz character of the CSD matrix}

HR-BL processing is based on the assumption that the CSD matrix of the original array is Toeplitz, which is a known property for a surface-noise-only field. ${ }^{19,22-24,28}$ However, measured CSD matrices do not always exhibit a Toeplitz structure. As an example, Fig. 4 shows the real and imaginary part of two CSD matrices obtained from the BOUNDARY-03 experiment ${ }^{11}$ by averaging 5-min segments collected about $40 \mathrm{~min}$ apart. Since the interest here is in the geometric structure of the matrices, rather than the values of their elements, to ease the comparison, each matrix has been normalized so that the maximum absolute value of its elements is 1. Two important differences are apparent. First, the CSD matrix in Figs. 4(a) and 4(b) does not show as clear a Toeplitz structure as the matrix in Figs. 4(c) and 4(d).

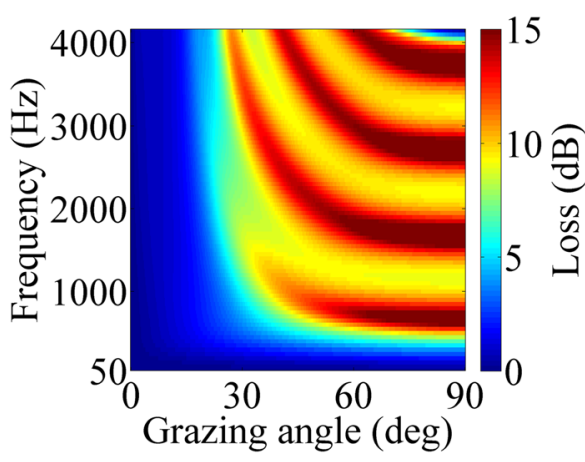

C

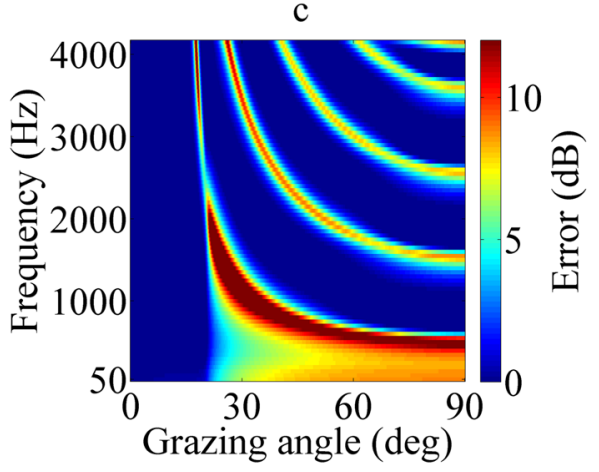

b

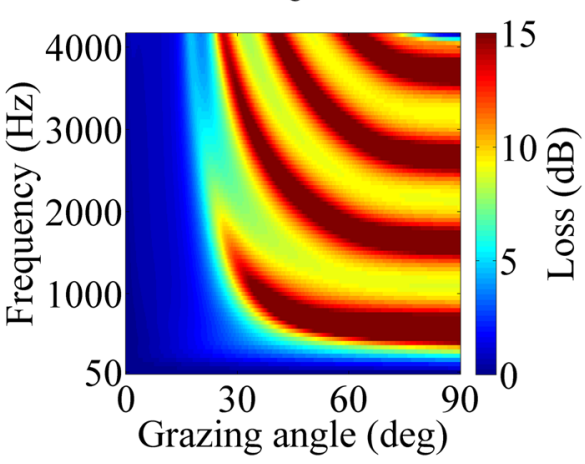

d

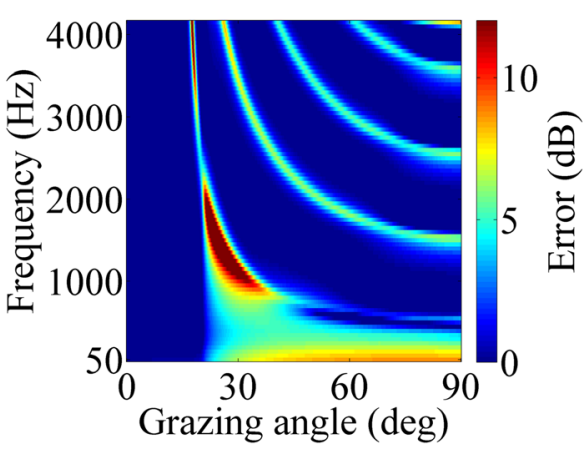

FIG. 3. (Color online) Estimated bottom loss and error over the frequency range $25-4166 \mathrm{~Hz}$ from OASN data for the environment in Table I. The bottom loss is estimated from the same $32 \times 32$ CSD matrix using a Hanning taper and CBF (a) and HR-BL processing (b). The error is computed as the pixel-bypixel difference in $\mathrm{dB}$ between the bottom loss predicted using the model by Jensen et al. (Ref. 1) and the bottom loss estimated by the CBF (c) and the HR-BL processor (d). 

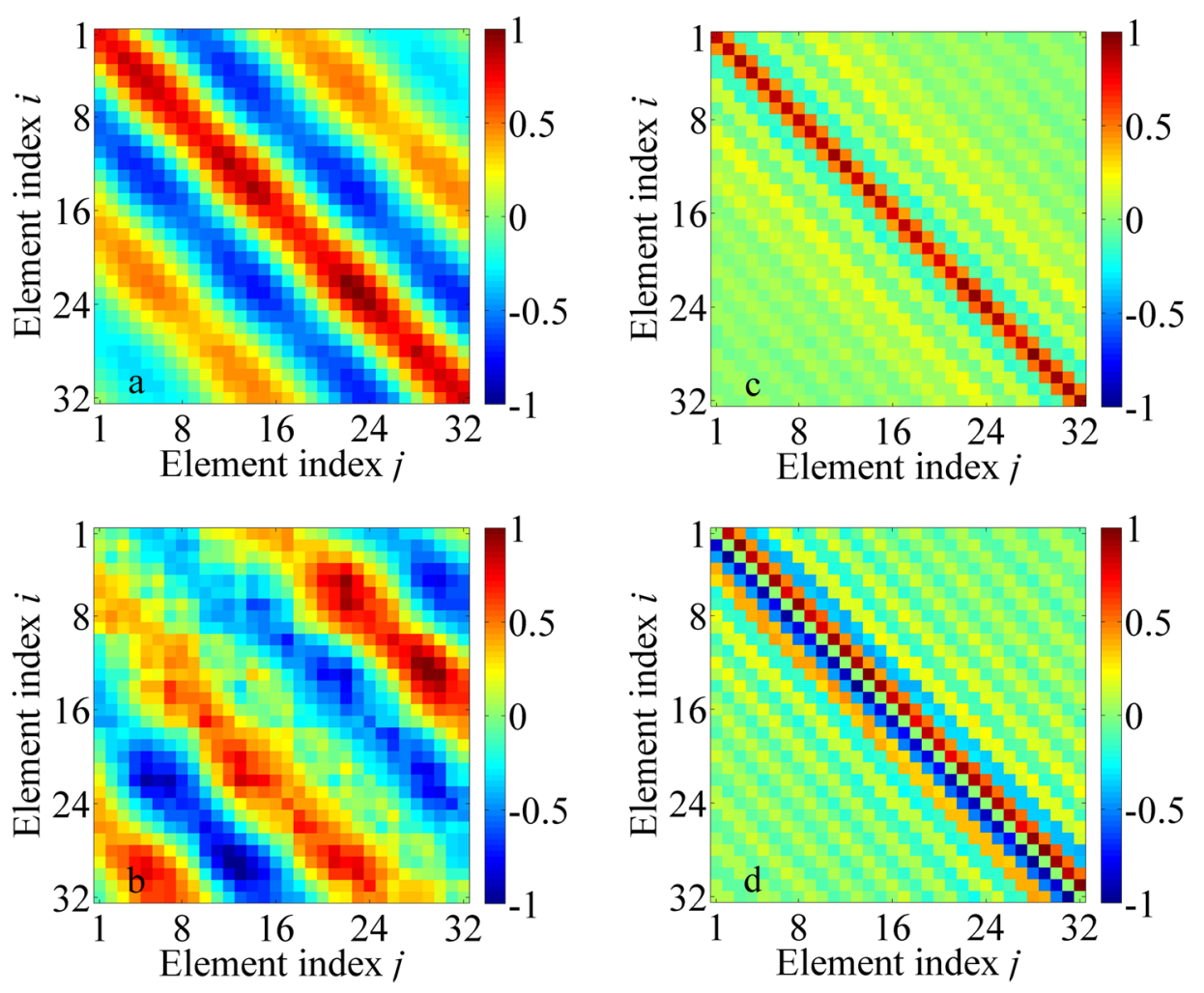

FIG. 4. (Color online) Real (top) and imaginary (bottom) parts of the normalized CSD matrices at $2156 \mathrm{~Hz}$, computed from two 5-min snapshots (collected about $40 \mathrm{~min}$ apart) from the BOUNDARY-03 experiment. The matrices in (c) and (d) appear to be closer to Toeplitz than the matrices in (a) and (b).

Second, its diagonal bands are wider, and do not decay as markedly when moving away from the main diagonal. These two differences appear to have a strong influence on the bottom loss estimated from these CSD matrices. The results shown in Fig. 5 were obtained by applying conventional beamforming and HR-BL processing to the $32 \times 32$ matrices shown in Fig. 4. In Fig. 5(a), both curves drop below zero at low grazing angles, an implausible result for a field generated only by surface noise and, therefore, an indication that some fundamental assumption in the model is violated. In this case, the HR-BL curve shows very large oscillations, which are inconsistent with the curves in Fig. 5(b) (from data collected about 40 min later), which shows more plausible curves: The physical-array curve appears to be a smoothed version of the HR-BL curve and the latter shows more marked oscillations and a higher bottom loss around endfire. In other words, the HR-BL processor results compare to the $\mathrm{CBF}$ results in a manner similar to what was observed for the OASN simulation (see Fig. 2).

The comparison between the two CSD matrices in Fig. 4 raises the question of what is inducing such dramatic alterations in the structure of the matrices. One possible cause of the non-Toeplitz character of the matrix is array deformation. Harrison's model for the spatial coherence function contains a term that is a function of the elevation angle of the line joining the two receivers. When the array is assumed to be perfectly vertical (as in this study), this term equals one and therefore does not appear in Eq. (11). But if the array is deformed, in general, the elevation angle of the line joining an arbitrary pair of sensors will vary depending on the particular pair chosen, changing the value of the additional term. This variation will reflect on the CSD matrix by introducing some variability along the diagonals. Given the arbitrary character of array deformation, it is hard to provide a systematic study of the influence of this effect on the CSD matrices. OASN simulations conducted with rather severe
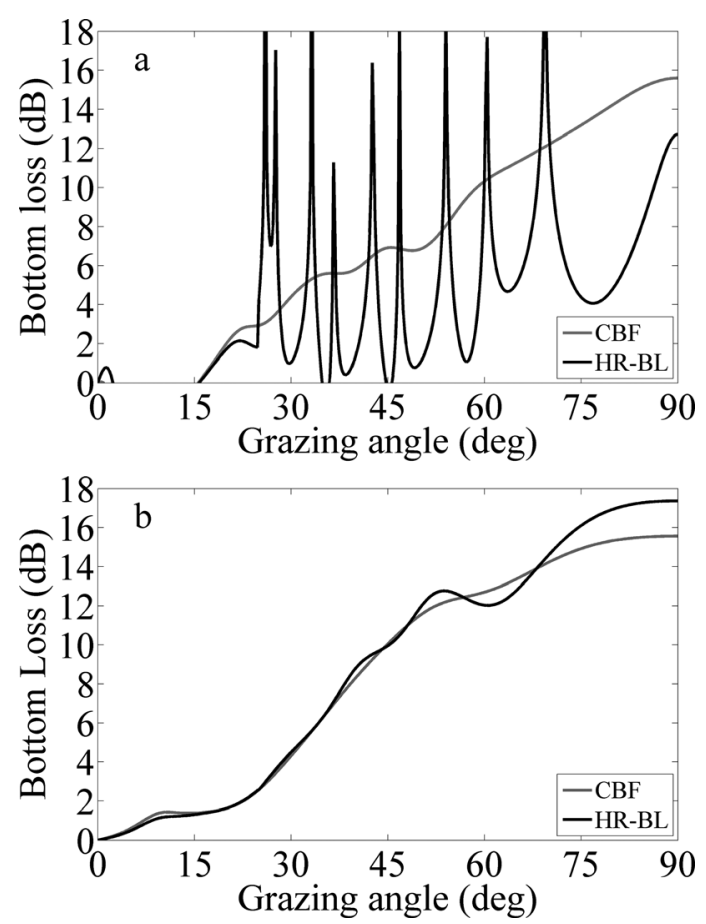

FIG. 5. BL curves: 32-element CBF vs HR-BL for the same data as in Fig. 4. The drop below zero of the BL curves visible below $20^{\circ}$ in (a) [corresponding to Figs. 4(a) and 4(b)] is an indication that some violation of the model assumptions is occurring in this 5-min average. The large oscillations of the HR-BL curve are also inconsistent with the curve from data collected about 40 min later (b) [corresponding to Figs. 4(c) and 4(d)], where the two techniques compare in a manner analogous to what was observed for the OASN simulation (see Fig. 2). 


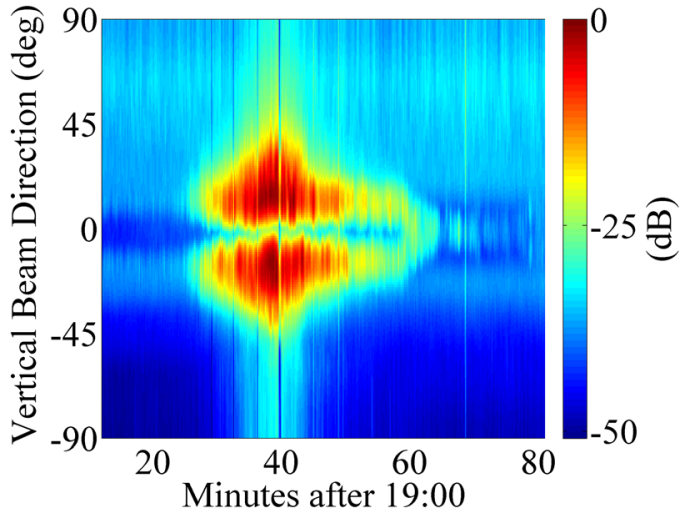

FIG. 6. (Color online) Beamformer output at $2156 \mathrm{~Hz}$, as a function of steering angle and time, from the dataset used to produce the CSD matrices in Fig. 4. A loud interferer appears close to broadside around time 19:25, and approaches the array reaching the closest point around time 19:40, as indicated by the broadening of the angle covered by the interferer. The CSD matrix shown in Figs. 4(a) and 4(b) corresponds to a 5-min time average centered around time 19:27:30 (when the interferer is quite strongly affecting the array output), while the matrix in Figs. 4(c) and 4(d) is based on an average centered around time 20:07:30 (when the interferer's influence is much reduced).

deformations showed increased variability along the diagonals, but not to the extent visible in Fig. 4, failing, in particular, to produce the alteration of the band structure.

It is reasonable to think that a nearby discrete source, such as a ship, could be responsible for the effects observed in Figs. 4 and 5; Fig. 6 seems to support this hypothesis by plotting the beamformer output at $2156 \mathrm{~Hz}$, as a function of steering angle and time. A loud interferer appears close to broadside around time 19:25, and approaches the array reaching the closest point around time 19:40, as indicated by the broadening of the angle covered by the interferer. The CSD matrix shown in Figs. 4(a) and 4(b) corresponds to a 5min time average centered around time 19:27:30 (when the interferer's presence is quite strongly affecting the array output), while the matrix in Figs. 4(c) and 4(d) is based on a time average centered around time 20:07:30 (when the interferer's influence is much reduced).

\section{RESULTS}

In this section, the procedures described in Sec. V for simulated data are applied to the CSD matrices obtained from data collected at sea by 32-element arrays during three separate experiments by the NATO-STO Centre for Maritime Research and Experimentation ${ }^{11,12}$ (CMRE-formerly NATO Undersea Research Centre). The data represent measurements from two different vertical arrays, at six different locations. The dataset identifiers used in this paper are
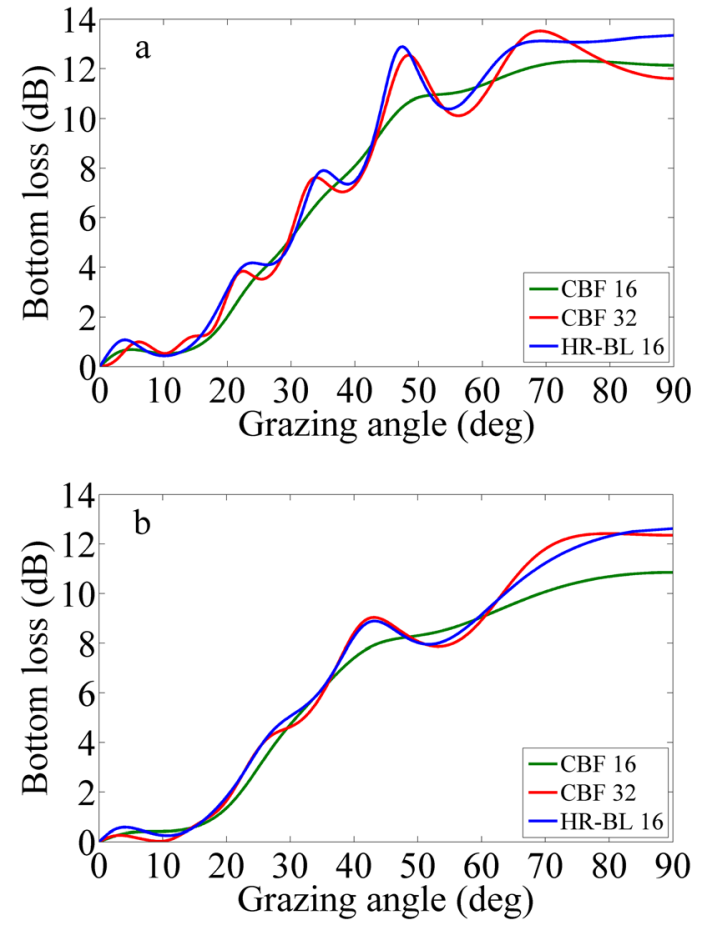

FIG. 7. (Color online) BL curves computed from two 5-min averages (data from the VLA-03 dataset) at $1313 \mathrm{~Hz}$ (a) and $972 \mathrm{~Hz}$ (b): Conventional beamforming $(\mathrm{CBF})$ for 32-element and 16-element physical array vs 16element HR-BL processor using a Taylor taper with $-30 \mathrm{~dB}$ sidelobe level. In both cases, the 16-element HR-BL processor reproduces the features of the 32-element CBF curve more faithfully than the 16-element CBF, and limits the BL disruption around endfire.

reported in Table II, together with the basic features of the array and acquisition system.

For the location of these measurements, the only ground truth available is in the form of normal incidence measurements (e.g., seismic chirp sonar), which can provide information about the layering of the bottom, but not the bottom loss, which is of interest in this paper. For this reason, Figs. 7-9 show two CBF lines, corresponding to BL estimates obtained using the full array (32 elements), and a sub-array composed of the first 16 elements. The third line, in each of the plots, is the bottom loss estimated by the HR-BL processor using data from the same sensors as the 16-element $C B F$. Since no BL ground truth is available, the estimate from the longer array is assumed to be the better one, and the performance of the 16-element HR-BL processor can be assessed by comparison with that of the two CBF results. All the CSD matrices were obtained by averaging $5 \mathrm{~min}$ of data. Both the HR-BL coherence function before the DFT, and the array data used by the CBF are tapered using a Taylor window with $-30 \mathrm{~dB}$ maximum sidelobe level (compared to the main lobe).

TABLE II. Datasets and array basic features—all deployments were drifting.

\begin{tabular}{lcccc}
\hline \hline Dataset identification & Number of elements & Spacing $(\mathrm{m})$ & Sampling frequency $(\mathrm{Hz})$ & Design frequency $(\mathrm{Hz})$ at $c=1500 \mathrm{~m} / \mathrm{s}$ \\
\hline MFA-03 & 32 & 0.18 & 12000 & 4166 \\
MFA-04 & 32 & 0.18 & 12000 & 4166 \\
VLA-03 & 32 & 0.50 & 6000 & 1500 \\
\hline \hline
\end{tabular}



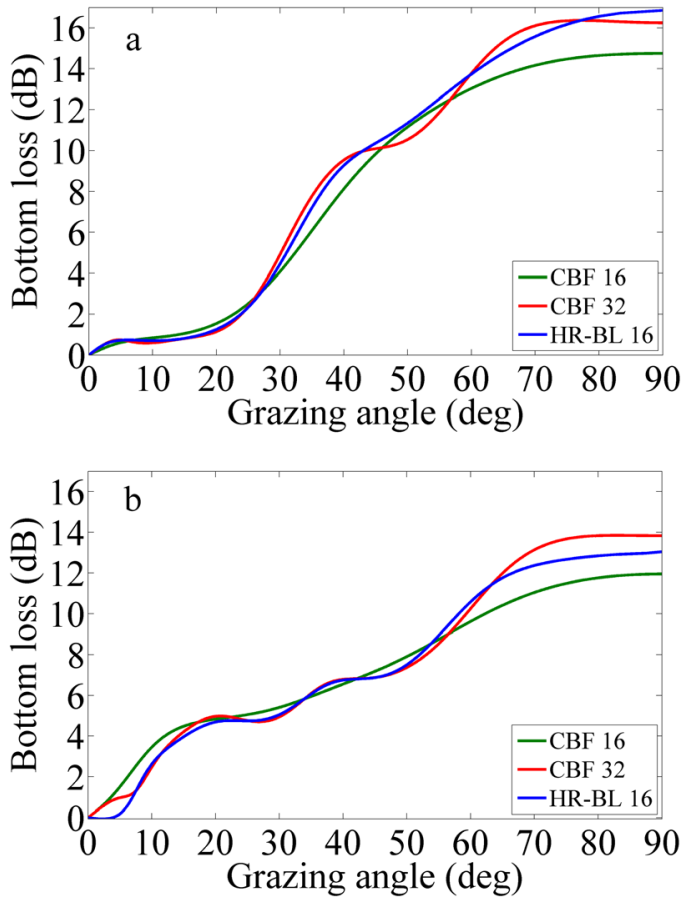

FIG. 8. (Color online) Bottom-loss curves computed from two 5-min averages (data from the MFA-03 dataset) at $2000 \mathrm{~Hz}$ (a) and $2250 \mathrm{~Hz}$ (b); processing and naming conventions are the same as in Fig. 7.

For the 16-element cases, the CBF curves show a marked degradation in angular resolution, in the form of less pronounced, wider peaks and valleys, and a generally lower loss estimated toward $90^{\circ}$. The HR-BL curves are obtained by processing only the first 16 elements of the array. The HR-BL curves appear largely immune to the degradation
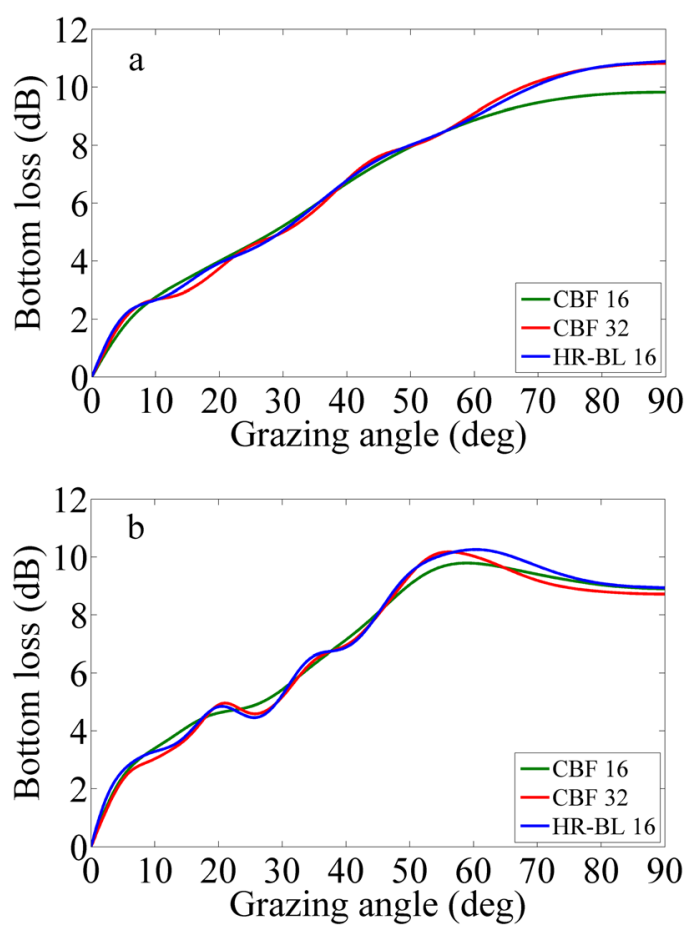

FIG. 9. (Color online) Bottom-loss curves computed from two 5-min averages (data from the MFA-04 dataset) at $2414 \mathrm{~Hz}$ (a) and $3070 \mathrm{~Hz}$ (b); processing and naming conventions are the same as in Fig. 7. experienced by the 16-element CBF, very closely resembling the performance of the 32-element CBF. Note that, given the larger inter-element spacing, the frequencies in the VLA-03 case are lower than in the others, but CBF and HR-BL compare in similar terms.

\section{CONCLUSIONS}

A previously introduced derivation in frequency-wave number domain of the bottom plane-wave power reflection coefficient from the array coherence function has been extended to include the effects of volume attenuation and variable sound speed in the water column. The main result is that, under certain conditions, for a surface-noise-only field, it is possible to obtain the reflection coefficient (and therefore the bottom loss) by computing the Fourier transform of the coherence function $C_{\omega}(z)$. A technique has been presented, and theoretically justified, that improves on the BL estimate provided by conventional beamforming by exploiting the Toeplitz structure of the noise-only CSD matrix and a DFT implementation of beamforming. The technique has been demonstrated both on simulated and measured data. When the estimated CSD matrix obtained from array data is sufficiently close to Toeplitz, experimental results show that a 16-element array can improve the estimated bottom loss, achieving an angular resolution comparable to that of a matrix-product implementation of CBF on a 32-element array.

\section{ACKNOWLEDGMENTS}

The authors gratefully acknowledge the support of the Office of Naval Research Ocean Acoustics Program (ONROA Code 322OA). We would also like to thank the NATOSTO Centre for Maritime Research and Experimentation (CMRE), Chris Harrison, and Peter Nielsen for providing the experimental data and valuable discussions. Finally, we would like to thank John Gebbie for pointing out the effective triangular windowing of the conventional beamformer when applied to the noise data.

${ }^{1}$ F. B. Jensen, W. A. Kuperman, M. B. Porter, and H. Schmidt, "Fundamentals of ocean acoustics," in Computational Ocean Acoustics (Modern Acoustics and Signal Processing), 2nd ed. (Springer, New York, 2011), Chap. 1, pp. 38-50.

${ }^{2} \mathrm{R}$. Hamson, "The modelling of ambient noise due to shipping and wind sources in complex environments," Appl. Acoust. 51, 251-287 (1997).

${ }^{3}$ E. L. Hamilton, "Geoacoustic modeling of the sea floor," J. Acoust. Soc. Am. 68, 1313-1340 (1980).

${ }^{4}$ E. L. Hamilton and R. T. Bachman, "Sound velocity and related properties of marine sediments," J. Acoust. Soc. Am. 72, 1891-1904 (1982).

${ }^{5}$ C. H. Harrison and D. G. Simons, "Geoacoustic inversion of ambient noise: A simple method," J. Acoust. Soc. Am. 112, 1377-1389 (2002).

${ }^{6}$ C. H. Harrison, "Sub-bottom profiling using ocean ambient noise," J. Acoust. Soc. Am. 115, 1505-1515 (2004).

${ }^{7}$ J. I. Arvelo, "Robustness and constraints of ambient noise inversion," J. Acoust. Soc. Am. 123, 679-686 (2008).

${ }^{8} \mathrm{M}$. Siderius and C. Harrison, "High-frequency geoacoustic inversion of ambient noise data using short arrays," AIP Conf. Proc. 728, 22-31 (2004).

${ }^{9}$ J. E. Quijano, S. E. Dosso, J. Dettmer, L. M. Zurk, M. Siderius, and C. H. Harrison, "Bayesian geoacoustic inversion using wind-driven ambient noise," J. Acoust. Soc. Am. 131, 2658-2667 (2012).

${ }^{10}$ M. Siderius, L. Muzi, C. H. Harrison, and P. Nielsen, "Synthetic array processing of ocean ambient noise for higher resolution seabed bottom loss estimation," J. Acoust. Soc. Am. 133, EL149-EL155 (2013). 
${ }^{11}$ C. H. Harrison, "Performance and limitations of spectral factorization for ambient noise sub-bottom profiling," J. Acoust. Soc. Am. 118, 2913-2923 (2005).

${ }^{12} \mathrm{C}$. H. Harrison and M. Siderius, "Bottom profiling by correlating beamsteered noise sequences," J. Acoust. Soc. Am. 123, 1282-1296 (2008).

${ }^{13}$ M. Siderius, C. H. Harrison, and M. B. Porter, "A passive fathometer technique for imaging seabed layering using ambient noise," J. Acoust. Soc. Am. 120, 1315-1323 (2006).

${ }^{14}$ P. Gerstoft, W. S. Hodgkiss, M. Siderius, C.-F. Huang, and C. H. Harrison, "Passive fathometer processing," J. Acoust. Soc. Am. 123, 1297-1305 (2008).

${ }^{15}$ S. L. Means and M. Siderius, "Effects of sea-surface conditions on passive fathometry and bottom characterization," J. Acoust. Soc. Am. 126, 2234-2241 (2009).

${ }^{16}$ M. Siderius, H. Song, P. Gerstoft, W. S. Hodgkiss, P. Hursky, and C. Harrison, "Adaptive passive fathometer processing," J. Acoust. Soc. Am. 127, 2193-2200 (2010).

${ }^{17}$ J. Traer, P. Gerstoft, and W. S. Hodgkiss, "Ocean bottom profiling with ambient noise: A model for the passive fathometer," J. Acoust. Soc. Am. 129, 1825-1836 (2011).

${ }^{18}$ D. H. Johnson and D. E. Dudgeon, "Arrays and apertures," in Array Signal Processing Concepts and Techniques (Prentice-Hall, Upper Saddle River, NJ, 1993), Chap. 3, pp. 65, 89.
${ }^{19}$ C. H. Harrison, "Formulas for ambient noise level and coherence," J. Acoust. Soc. Am. 99, 2055-2066 (1996).

${ }^{20}$ C. H. Harrison, "Noise directionality for surface sources in rangedependent environments," J. Acoust. Soc. Am. 102, 2655-2662 (1997).

${ }^{21}$ H. Schmidt, OASES Version 3.1 User Guide and Reference Manual (Massachusetts Institute of Technology, Cambridge, MA, 2004).

${ }^{22}$ W. S. Liggett and M. J. Jacobson, "Noise covariance and vertical directivity in a deep ocean," J. Acoust. Soc. Am. 39, 280-288 (1966).

${ }^{23}$ D. R. Barclay and M. J. Buckingham, "Depth dependence of wind-driven, broadband ambient noise in the Philippine Sea," J. Acoust. Soc. Am. 133 62-71 (2013).

${ }^{24}$ M. J. Buckingham, "A theoretical model of ambient noise in a low-loss, shallow water channel,” J. Acoust. Soc. Am. 67, 1186-1192 (1980).

${ }^{25}$ F. B. Jensen, W. A. Kuperman, M. B. Porter, and H. Schmidt, "Normal modes," in Computational Ocean Acoustics (Modern Acoustics and Signal Processing), 2nd ed. (Springer, New York, 2011), Chap. 5, p. 340.

${ }^{26}$ B. F. Cron and C. H. Sherman, "Spatial-correlation functions for various noise models," J. Acoust. Soc. Am. 34, 1732-1736 (1962).

${ }^{27}$ B. F. Cron, "Addendum: Spatial-correlation functions for various noise models," J. Acoust. Soc. Am. 38, 885 (1965).

${ }^{28}$ H. Cox, "Spatial correlation in arbitrary noise fields with application to ambient sea noise," J. Acoust. Soc. Am. 54, 1289-1301 (1973). 